\title{
Analysis on Key Ideas of Research Progress in Biotechnology Crop Food Detection Technology
}

\author{
Dong Tianfei \\ Chifeng Industrial Vocational Technical College, Chifeng City, Inner Mongolian Autonomous Region, China, 024000
}

\begin{abstract}
Food not only provides nutrition to people, maintains vitality, but also it is related to people's health. In view of the fact that current food safety issues are still common, it is very important to do a good job in food safety testing. Biotech crop food is a product that can be directly consumed. In order to improve people's trust in such products, scientific biotechnology testing methods can improve the efficiency and quality of food testing and ensure people eat food safely. This paper will analyze the basic types of food testing technology, and then introduce the application of several key detection technologies in biotech crop food testing, and discuss the shortcomings in current food testing, and do a brief overview of future development on the biotech crop food testing technology.
\end{abstract}

\section{Biotech Crop Food Overview}

Biotechnology crop food is a kind of food that is processed by biotech crops as direct food or raw materials. At present, many countries have carried out a lot of development and research work, and the biotechnology is promoted and applied. Technical crops have entered the stage of large-scale commercial production. In China, the area of biotechnology crops has exceeded 3.7 million $\mathrm{hm} 2$. In order to promote the development of this technology and the marketization of biotech crop foods, China has promoted several major scientific and technological projects to make biotech rice, corn and other crops realized the commercialization. However, while bringing social and economic benefits to the country, it has also brought many problems. In particular, biotech crop foods have not yet been scientifically demonstrated in terms of human health and environmental safety. People also eat biotech crop foods. There are concerns, and it is difficult to promote large-scale promotion.

In view of the fact that biotech crop foods can be directly consumed, in the new era of frequent food safety issues, the quality inspection and management of bio-foods must be strengthened. Therefore, biotech crop products must undergo strict quality inspection and label management. This will increase consumer trust. Many countries have also carried out the necessary testing and monitoring of biotech crop foods, and established first-class testing institutions and standardized testing systems with internal quality control to meet the needs of biotech crop food testing. China has also established standardized biotechnology crop food testing laboratories, and some biotechnology research and development companies have also established cooperation with certain international or regional accredited institutions. Although there is no uniform standard in the detection method, the specific detection technology includes nucleic acid detection technology and protein detection, which is suitable for the detection of biotechnology crop raw materials and biotechnology crop production and processing food.

\section{Analysis of Basic Types of Food Testing Technology}

\subsection{Bioenzyme and Biochip Technology}

Biochip is a new technology for detecting food safety. It has informatization characteristics and can be fully applied to food testing projects by means of photosynthetic synthesis. This technology can judge the most basic food quality, so the detection application range is wide and the detection speed is fast. For large-scale food detection, this technology can be completed with high efficiency and high quality. For foods with high quality requirements or import and export foods, biochip technology can ensure food detection results because of the high precision of food quality testing. In addition, bio-enzyme detection technology is also a highly accurate and efficient technology with a wide range of applications and higher sensitivity. This technology belongs to the enzyme-linked immunoassay. It is a new food detection technology based on immunology. It mainly detects micro-organisms in food and trace pollution caused by toxic pesticides.

\subsection{Polymerase Chain Reaction Detection}

Polymerase chain reaction detection technology, also 
known as PCR technology, has the advantages of both micro-detection and accuracy detection. It is based on molecular biology and uses DNA denaturation and renaturation principles to accurately detect in vitro. Amplification of the gene can detect a trace amount of palladium sequence, which can be divided into qualitative PCR and quantitative PCR. With the continuous development of biotechnology, technologies such as nested PCR, reverse PCR and thermal asymmetric interlaced PCR have been derived. Over the years, with the deeper understanding of the genetic characteristics of microorganisms, people have been able to identify the root causes of certain hereditary pathogens. PCR technology can be used to detect food contamination and specific genes caused by microorganisms. With the amplification processing of various types of biological genes, the detection sensitivity is further improved, so the application range is wide and has a very broad development space.

\subsection{Protein Detection Technology}

Protein detection utilizes the principle of immunochemistry to prepare antibodies for proteins expressed by foreign genes, and then combines the specific binding of antigen-antibodies and the efficient catalytic principle of enzymes on substrates to achieve detection of target proteins. Specifically, it includes an enzyme-linked immunosorbent assay, a test strip, and a Western hybridization technique. Among them, the enzyme-linked immunosorbent assay utilizes a kit, which has the advantages of quantitative and qualitative analysis, simple detection operation, and high degree of automation. By this method, it is possible to detect Kangglyphosate soybean by the specific binding of the antibody to the protein in the food, and the detection sensitivity is higher than $0.1 \%$. The test strip method can detect the lowest value of protein, and has the advantages of rapid and high efficiency, easy operation and portable. Currently, detection reagents for detecting herbicide-resistant shikimate acetyltransferase and insect-resistant $\mathrm{Bt}$ toxic protein have been developed and test strips.Western hybridization uses the detection technology in plant genetic engineering to detect proteins in foreign genes. This method requires membrane transfer and hybridization. The operation is complicated and costly, and it is not suitable for the detection of large-scale food samples. The disadvantages of this method are the following. First, because the expression of the foreign gene is tissue-specific, the protein expressed by the inducible gene cannot be detected in a specific material; the second protein is denatured during processing, so it is not suitable for processing. It is a test for deep-processed food products and can only be used in the detection of raw materials and the detection of processed foods. Thirdly, it is necessary to prepare specific antibodies, and the preparation is complicated, so the application range of the technology is not very extensive.

\subsection{Sensor Detection Technology}

Sensor detection technology is used to identify the biological characteristics of food, enter these biometrics into the identification system and then achieve the corresponding transformation. From the whole process of food testing, the sensing technology can run through the whole process of food production and processing, and can complete high-precision detection in a relatively fast time, obtain accurate detection information, and obtain the entire inspection process optimization.

\subsection{Near Infrared Spectroscopy}

The near-infrared spectrum is between the visible region and the infrared region, and the wavelength ranges from 0.75 to $2.5 \mathrm{um}$. By using near-infrared spectroscopy, characteristic information of hydrogen-containing groups of all organic molecules in the sample can be obtained, and a plurality of organic molecules can be detected simultaneously. Near-infrared light enables the penetration of substances without pre-treatment of the sample, and does not cause any harm to the human body, does not cause any pollution to the environment, and has the advantages of rapid and efficient detection. The technology is widely used in the detection of agricultural products, and the technology is very mature, but it is still relatively rare in the detection of biotech agricultural products. At present, experiments have shown that the near-infrared spectroscopy can be used to separate RRS from common non-biotech soybeans with an accuracy of $84 \%$. In addition, the BP network recognition model can be used to identify and analyze 24 transgenic Bt corns. When the absolute error is less than 0.05 , the detection accuracy of the detected samples can be $100 \%$. Because NIR spectroscopy cannot detect specific compounds, it cannot be used to accurately detect changes in DNA or a certain protein in biotechnology products. Therefore, the application in biotech crop food testing has great limitations.

\subsection{Electrochemiluminescence Detection Technology}

The technique refers to applying a voltage on an electrode, and then directly or indirectly generating chemiluminescence by using an electrochemical reaction, and the electrochemiluminescence has no radioactive hazard, and is a specific chemiluminescence caused by an electrochemical reaction on the electrode surface, and the sensitivity is high. Moreover, the detection linear range is wider and the detection speed is faster, and the general detection time is only tens of seconds to several minutes. Combining this technology with PCR technology and dual-probe hybridization technology can successfully determine whether a sample contains biotechnological components. The method needs to combine technologies such as PCR, so the operation steps of the method are relatively complicated and costly, and there are certain limitations in the detection of biotech crop foods. 


\section{Application of Food Testing Technology in Biotechnology Crop Food}

\subsection{Application of PCR Detection Technology}

This technology is very commonly used in food testing. In the application, by enriching microbial samples and detecting DNA sequences by means of cloning technology in polymerization, polymerase reaction decomposition can improve the polymerization ability in the detection, thereby ensuring Food safety. Compared with traditional microbial detection technology, this technology has higher automation degree and higher detection efficiency, thus avoiding the drawbacks of traditional microbial detection technology taking a long time, and can effectively improve the detection speed and detection accuracy. At this stage, the technology is mainly used to detect Salmonella, Escherichia coli and Staphylococcus aureus.

\subsection{Application of Enzyme-linked Immunosorbent Assay}

The enzyme-linked immunosorbent assay (ELISA) is based on the high specificity of the antigen-antibody reaction and the catalysis of the enzyme. The antigen is reacted with the enzyme standard in the sample to produce a complex, and then the color is developed, and then the colored substance is quantitatively and qualitatively analyzed. The technology is highly specific, with high sensitivity, high accuracy, high sample throughput and good reproducibility. Currently, this technology is used in the detection of food organophosphorus, chlorine pesticide residues and biotoxins.

\subsection{Application of Biosensor Technology}

Biosensor technology combines biological, chemical, physical, and informational content. After analyzing and tracking the measurable material, the biosensor is used to make the detected substance enter the substance sensitive substance, and then the chemical transducer is used after molecular identification. The physical transducer converts the information formed by the biological reaction into an electrical signal, and detects the detected substance after the amplification output. The technology is highly efficient and can quickly analyze a large amount of molecular information, and is mainly used to detect sugar substances such as fructose and maltose in foods. The technology can also use indicators to supervise and control the entire production process, and can provide good and comprehensive information for enterprise food production.

\subsection{Application of Biochip Technology}

The biochip technology utilizes optical and micro-spotting, so that the bio-analytical rules are regularly arranged on the surface of the glass piece, the nylon film, etc., and after forming the two-dimensional molecule, it is hybridized with the target molecule, and the hybridization signal is collected by a specific instrument, and then according to the corresponding rapid detection analysis of strength. The biochip also includes a computer chip and a chip lab, which can obtain a large amount of biological information conversion, and pays attention to the curing process of the chip detection technology in the technology, which can effectively improve the control capability of the entire detection technology. Biochip technology can also detect nutrients and pathogenic microorganisms in genetically modified foods and foods, and can quickly detect unsafe factors in foods, thereby ensuring food safety.

\subsection{Application of Gene Probe Detection Technology}

The gene probe detection technology can timely process the corresponding monitoring technology by copying and analyzing the DNA sequence, and can optimize the entire technology control. Corresponding detection elements are monitored through analysis of key points in technical control. The application of this technology can improve the accuracy of food monitoring and ensure the safety of food.

\section{Analysis of Problems and Future Development in Biotech Crop Food Testing}

If the biotech crop food detection technology is classified, it can be divided into two major categories: nucleic acid detection method and protein immunoassay. At present, these two methods have been applied in biotech crop food testing and have obtained corresponding research and application. The results, but in fact, because biotech crop foods are still in the initial stage of development, marketization, commodification is not high, and acceptance needs to be improved, so the corresponding technologies also have certain limitations in their detection. For example, PCR detection technology is suitable for the detection of biotechnological components in bioprocess crop foods. If it is used in deep processing food testing, it still faces certain challenges. It is mainly impossible to determine whether the raw material DNA can be accurately and efficiently extracted from many deep processing organisms. Extracted from technical crop products. In summary, the problem with nucleic acid detection in biotech crop food testing is mainly the ability to efficiently and accurately obtain high quality nucleic acids. At present, this problem requires purification of various inhibitors contained in different deep processing products, and purification and enrichment of small fragments and low-level DNA, such as DNA that has been developed and applied and the extraction kit can solve these problems to some extent. For protein immunoassay technology, the main drawback is that it lacks self-developed detection reagents, so the detection range of this method is limited, and deep 
processing will denature proteins, affecting the sensitivity and accuracy of detection, and can only be used at the beginning of testing the biotech ingredients for processed foods and raw materials.

\section{Conclusion}

In summary, the safety of biotech crop foods directly affects people's health. Therefore, it is necessary to strengthen the research and application of food testing technology for biotech crop foods. It requires fast, accurate and practical testing, and requires operation. Simple, not too cumbersome. At present, biotechnology crop detection technology is moving toward the development of kits. If ordinary people can purchase the reagent strips or kits they need to carry on the market, they can easily detect the food they purchased. Whether food contains biotech ingredients, but also we need to ensure the safety of the foods you eat.

\section{References}

1. Gao Juan,Jiang Huiying.Application of Bioassay Technology in Food Inspection[J].Food Safety Guide,2017(06):76.

2. Wang Peng. The latest application of biotechnology in food safety testing [J]. Food Safety Guide, 2017 (03): 19.

3. Liu Sai. Application of Biotechnology in Food Testing [J]. Food Safety Guide, 2017 (03): 65.

4. Zhao Miaomiao. Application of Biotechnology in Food Testing [J]. Food Safety Guide, 2017 (03): 111. 